\title{
THE ISOCHRONOUS CYCLOTRON AND HIGH-RESOLUTION NUCLEAR SPECTROSCOPY*
}

\author{
W. C. PARKINSON and J. BARDWICK \\ Cyclotron Laboratory, Department of Physics, The University of Michigan, Ann Arbor, Michigan 48104, U.S.A.
}

Received 16 September 1969

The practical limitations on high-resolution charged-particle spectroscopy imposed by the size and brightness of the effective source from an accelerator are discussed. Methods of measuring the characteristics of the source, and the parameters of the ion-

\section{Introduction}

The new generation of cyclotrons, with their higher energies and relatively good emittance has permitted a significant improvement in the information obtained from charged-particle nuclear spectroscopy. High resolution spectroscopy requires in addition to the cyclotron as the source of particles, a "beam preparation" system consisting of dispersive elements and slits, a target, a reaction-products analysis system and particle detectors. Practical limitations on resolution are imposed by the size and the brightness of the source, the quality of the ion-optical elements and a variety of kinematic effects. The quality of the dispersive elements and the slit system together with the size of the source conspire to determine the line-shape and therefore the ultimate resolution, and these are discussed in section 2. Methods of measuring the characteristics of the source and the parameters of the ion-optical system are discussed in sections 3 and 4, while section 5 contains some general remarks on resolution and brightness as applied to isochronous cyclotrons. Section 6 describes our method of momentum calibration of the spectrometer.

\section{Line shape and resolution}

Consider the beam preparation system ${ }^{1}$ ) shown in fig. 1. The cyclotron provides an effective source (S), the two magnets $P_{1}$ and $P_{2}$ are the dispersive elements, and slits $W_{1}$ and $w_{2}$ are placed at the image surfaces of $P_{1}$ and $P_{2}$. It is the beam through the slit $w_{2}$ at the focal surface of $P_{2}$ which impinges on the target.

For the system shown it is the radial dispersion that is of interest and follwing $\left.\mathrm{Judd}^{2}\right)$ it is defined as $D^{\prime}=\left(\Delta x / r_{0}\right) /(\Delta p / p)=(1+M) /(1-n)$ where $\Delta x$ is the displacement perpendicular to the optic axis for a change in momentum $\Delta p, r_{0}$ is the mean radius of curvature, $p$ is the central momentum, $n=-(r / B) \times$ $\times(\mathrm{d} B / \mathrm{d} r)$ is the field index, and $M$ is the radial magni-

* Supported in part by the U.S. Atomic Energy Commission. optical system are described. Results of such measurements with an isochronous cyclotron as the source, are given. The technique for calibration of the spectrometer is described.

fication. For our purposes it is more convenient to use a quantity which we shall also call the dispersion defined by $D=2[(1-n) /(1+M)]\left(E_{0} / r_{0}\right)$, where $E_{0}$ is the particle energy corresponding to $r_{0}$. Thus for magnets with $n=\frac{1}{2}, M=1, r=2 \mathrm{~m}$ and $E_{0}=$ $=40 \mathrm{MeV}, D=10 \mathrm{keV} / \mathrm{mm}$, whereas $D^{\prime}=4$.

The resolving power is defined using as the criterion the fractional change in energy required to move an image out of a slit of width $w$ so that it will not be confused with an adjacent image, thus $R=E / \Delta E=$ $=D^{\prime} r_{0} /(2 \Delta x)=[(1+M) /(1-n)]\left(r_{0} / 2 w M\right)$, where $\Delta x=M w$. If $n=\frac{1}{2}, M=1, r_{0}=2 \mathrm{~m}, w=1 \mathrm{~mm}$, then $R=4000$.

Assume now that the particles extracted from the cyclotron can be considered as emerging from some effective source of radial width $S$, and that particles of all energies are emitted from each element of $S$ and that $N$, the number of particles per unit time per unit $\Delta E$, is constant. In considering the effect of $S$ on the resolution and line shape we consider first the ideal

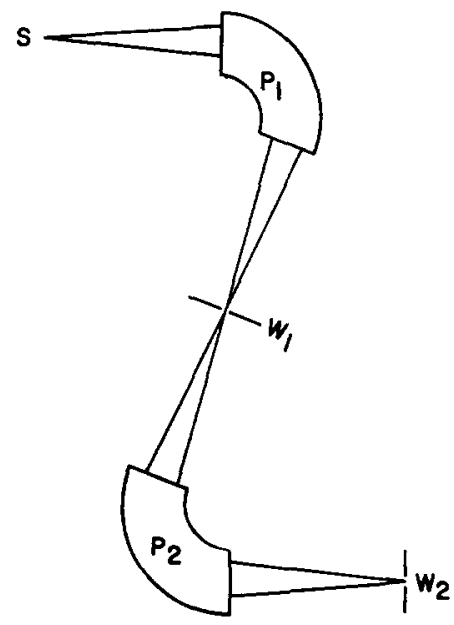

Fig. 1. Schematic of the beam preparation system at The University of Michigan 83-inch cyclotron. Note that the beam extracted from the cyclotron enters the first dispersing element without passing through a defining slit. 

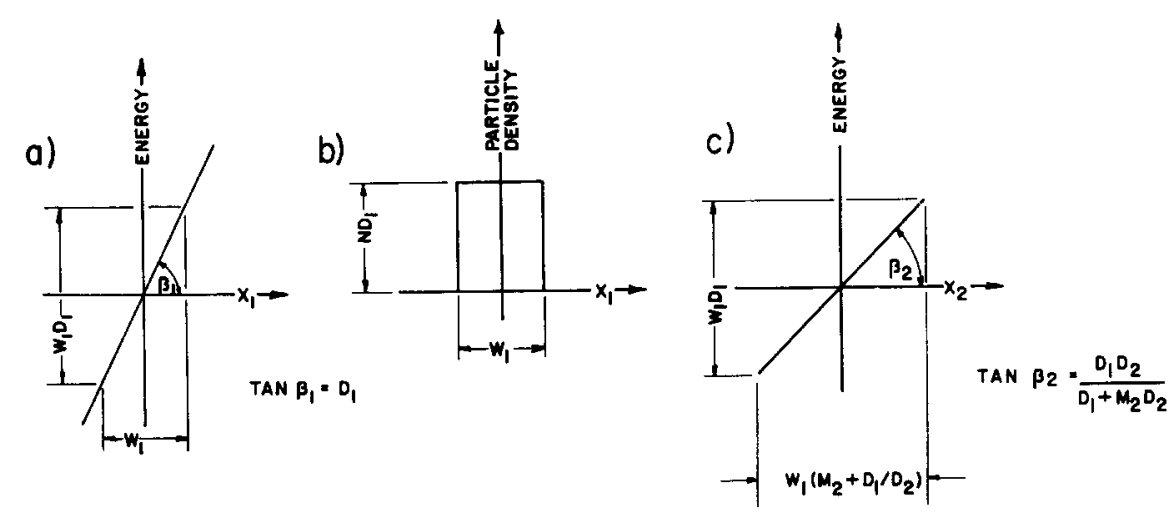

Fig. 2a. Energy as a function of position at $x_{1}$; b. intensity at $x_{1}$; c. energy vs position at $x_{2}$.

case $S=0$, then the case of an incoherent source of width $S$, and finally a coherent source of width $S$.

\subsection{IDEALIZED LINE SOURCE $(S=0)$}

At the image surface of the first magnet $P_{1}$ the energy of the particles as a function of their distance $x$, measured normal to the optic axis is $E=E_{0}+x_{1} D_{1}$ $=E_{0}+x_{1} \tan \beta_{1}$ where $D_{1}=\mathrm{d} E / \mathrm{d} x_{1}=\tan \beta_{1}$ and the maximum and minimum energies through a slit of total width $w_{1}$ placed there are $E_{\mathrm{m}}=E_{0} \pm\left(w_{1} / 2\right) D_{1}$ so that the total spread in energy is $\Delta E=w_{1} D_{1}$. The number of particles through each element $\Delta x_{1}$ is $N D_{1}$.

Each element at position $x_{1}$ within $\mathrm{w}_{1}$ images at the focal surface of $\mathrm{P}_{2}$ at the position $x_{2}=x_{1} M_{2}+$ $+x_{1} D_{1} / D_{2}$ and since the energy of the particles passing through $x_{2}$ is the same as through $x_{1}$,

$E_{2}=E_{1}=E_{0}+x_{1} D_{1}=E_{0}+x_{2} D_{1} D_{2} /\left(M_{2} D_{2}+D_{1}\right)$.

The number of particles through each element of $x_{2}$ is $N D_{1} /\left(M_{2}+D_{1} / D_{2}\right)$ and the total number through $\mathrm{w}_{2}$ is $N D_{1} w_{2} /\left(M_{2}+D_{1} / D_{2}\right)$. These relations are illustrated in fig. 2. Note that if $w_{2} \leqslant w_{1}\left(M_{2}+D_{1} / D_{2}\right)$, the spread

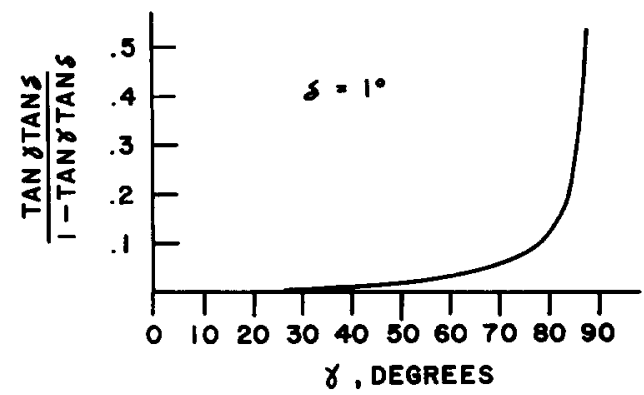

Fig. 3. Effect of tilting the focal surface. in energy of the particles through $\mathrm{w}_{2}$ is $\Delta E_{2}=w_{2}$ $\tan \beta_{2}=w_{2} D_{1} D_{2} /\left(D_{0}+M_{2} D_{2}\right)$.

However, if the image surface is not normal to the optic axis, then the energy spread through a radial slit will be increased by a factor $[1+\tan \gamma \tan \delta /(1-\tan \gamma$ $\tan \delta)]$ where $\gamma$ is the angle at which the image surface deviates from normal, and $\delta$ is half the angular spread in the beam at the location of the slit. The function $\tan \gamma \tan \delta /(1-\tan \gamma \tan \delta)$ is plotted against $\gamma$ in fig. 3. For the usual case of a few degrees divergence in the beam, the loss in resolution becomes significant only if the image surface is tilted at more than $70^{\circ} *$.

\subsection{INCOHERENT SOURCE OF WIDTH $S$}

If the source is incoherent, so that every element of S emits particles of all energies, the spread in energy through any element $\mathrm{d} x_{1}$ of slit $\mathrm{w}_{1}$ is $\Delta E_{x_{1}}=S M_{1} D_{1}$ and the number of particles through each $\mathrm{d} x_{1}$ is independent of $x_{1}$, and depends only on $S, M_{1}$ and $D_{1}$ and is proportional to $S$. This is illustrated in fig. 4a.

An element at position $x_{1}$ images on $\mathrm{P}_{2}$ at $x_{2}$ $=x_{1} M_{2}+x_{1} D_{1} / D_{2}$ and because it emits particles of $\Delta E_{x}=S M_{1} D_{1}, x_{2}$ will have a spatial extent corresponding to $S M_{1} D_{1} / D_{2}$; thus $x_{2}=x_{1}\left(M_{2}+D_{1} / D_{2}\right) \pm$ $\pm(S / 2) M_{1} D_{1} / D_{2}$. For any value of $w_{1}$ the total extent along the image surface (taken as perpendicular

\footnotetext{
* In reaction products analysis systems, there usually is no loss in resolution due to the angle of the focal surface since the particle detectors are placed along the surface. However, in beam handling systems which accept only a small range of momenta and have slits normal to the optic axis, the resolution may be seriously degraded. The use of multiple elements in such systems, particularly quadrapoles with their inherent property of momentum dispersion along the optic axis may, depending on the design of the individual system, tend to enhance this effect.
} 


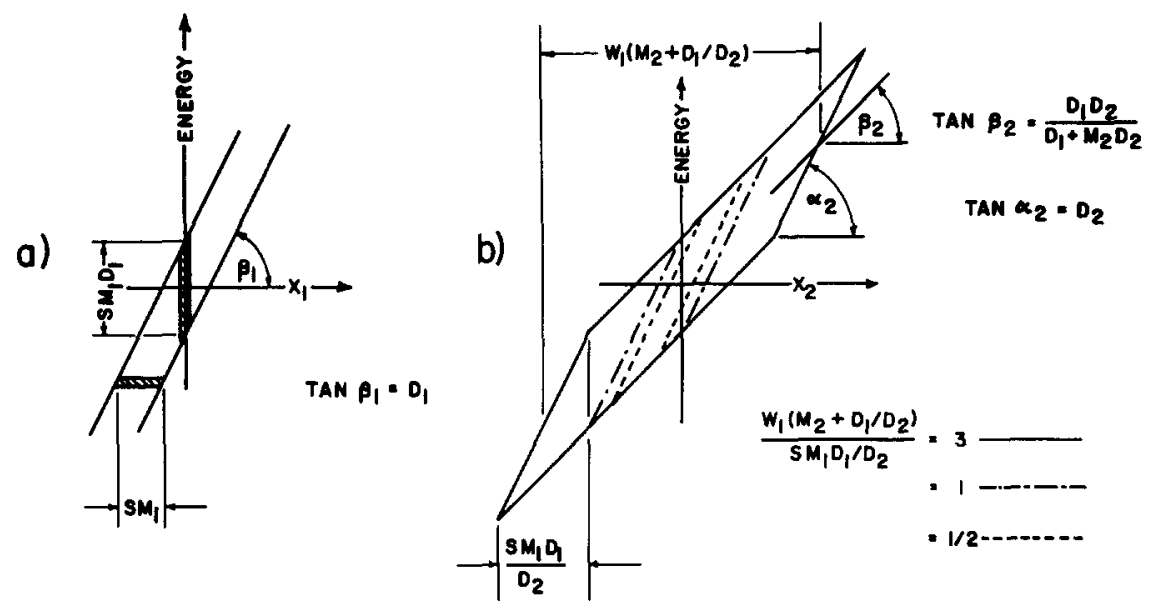

Fig. 4. Energy vs position for a finite source; a. at $x_{1}$, b. at $x_{2}$.

to the optic axis) of $\mathrm{P}_{2}$ is

$$
X=w_{1}\left(M_{2}+D_{1} / D_{2}\right)+S M_{1} D_{1} / D_{2}
$$

To move the image $X$ across a slit $w_{2}$ (by changing the magnetic field of $\left.\mathrm{P}_{2}\right)$ it must move a distance $\left(X+w_{2}\right)$ which is equivalent to an energy change $\left(X+w_{2}\right) D_{2}$ or $\Delta E_{2}$ equi vaient $=\left[w_{1}\left(D_{1}+M_{2} D_{2}\right)+w_{2} D_{2}+S M_{1} D_{1}\right]$. Note that in the limit of $w_{1}=0$ there is spatial spread across $x_{2}$ given by $X=S M_{1} D_{1} / D_{2}$ with energy spread $S M_{1} D_{1}$.

For the case $S=0$, the energy as a function of $x_{2}$ is given by the equation of a straight line through the origin $\left(E=E_{0}\right)$ of slope $D_{1} D_{2} /\left(D_{1}+M_{2} D_{2}\right)=\tan \beta_{2}$. As the width of $S$ is "turned on" the width of the image about each point on $x_{2}$ is given $S M_{1} D_{1} / D_{2}$ and there is a corresponding linear energy spread of $S M_{1} D_{1}$. The slope of the source line is $\tan \alpha_{2}=D_{2}$. At any given $x_{2}$ the source contributes an energy spread for $S \leqslant 2 w_{1}$ of $\Delta E_{\mathrm{S}}=S M_{1} M_{2} D_{1} D_{2} /\left(D_{1}+M_{2} D_{2}\right)$. The spatial extent and spread in energy along $x_{2}$ is

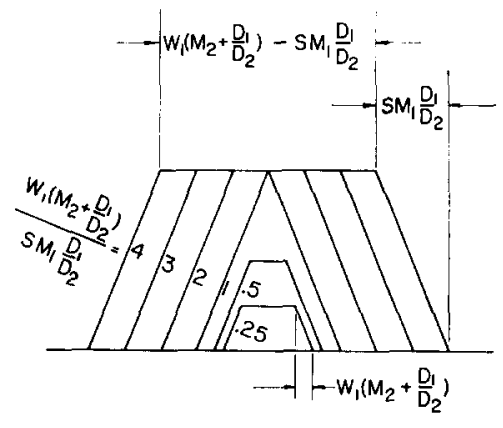

Fig. 5. Intensity (ordinate) as a function of position along $x_{2}$ for several values of $w_{1} / S$. illustrated in fig. $4 \mathrm{~b}$ for various ratios of $w_{1}$ and $S$. The relative intensity along $x_{2}$ is shown in fig. 5 .

The total energy spread across the image surface of $\mathrm{P}_{2}$ is $\Delta E_{2}$ total $=\left(S M_{1}+w_{1}\right) D_{1}$. At any particular value of $x_{2}$ there is a spread in energy corresponding to $E_{2}=E_{0}+\left[D_{1} D_{2} /\left(D_{1}+M_{2} D_{2}\right)\right]\left[x_{2} \pm(S / 2) M_{1} M_{2}\right]$. Slits of width $w_{2}$ placed at the focal surface of $\mathrm{P}_{2}$ may limit the spread in energy of the particles striking the target. In terms of the parameters of the system, the energy spread of the beam on the target is given by:

Case 1: $\left[S M_{1} D_{1} / D_{2}\right] /\left[w_{1}\left(M_{2}+D_{1} / D_{2}\right)\right] \leqslant 1$.

a. If $0 \leqslant w_{2} \leqslant w_{1}\left(M_{2}+D_{1} / D_{2}\right)+S M_{1} D_{1} / D_{2}$, then $\Delta E_{t}=\left(w_{2}+S M_{1} M_{2}\right) D_{1} D_{2} /\left(D_{1}+D_{2} M_{2}\right)$;

b. if $w_{2} \geqslant w_{1}\left(M_{2}+D_{1} / D_{2}\right)+S M_{1} D_{1} / D_{2}$, then $\Delta E_{t}=\left(w_{1}+S M_{1}\right) D_{1}$.

Case 2: $\left[S M_{1} D_{1} / D_{2}\right] /\left[w_{1}\left(M_{2}+D_{1} / D_{2}\right)\right] \geqslant 1$.

a. If $w_{2} \leqslant S M_{1} D_{1} / D_{2}-w_{1}\left(M_{2}+D_{1} / D_{2}\right)$, then $\Delta E_{t}=\left(w_{1} M_{2}+w_{2}\right) D_{2}$;

b. if $S M_{1} D_{1} / D_{2}+w_{1}\left(M_{2}+D_{0} / D_{2}\right) \geqslant w_{2} \geqslant$ $\geqslant S M_{1} D_{1} / D_{2}-w_{1}\left(M_{2}+D_{1} / D_{2}\right)$, then $\Delta E_{t}=\left(w_{2}+S M_{1} M_{2}\right) D_{1} D_{2} /\left(D_{1}+M_{2} D_{2}\right)$;

c. if $w_{2} \geqslant w_{1}\left(M_{2}+D_{1} / D_{2}\right)+S M_{1} D_{1} / D_{2}$, then $\Delta E_{t}=\left(w_{1}+S M_{1}\right) D_{1}$.

For a system with at least two dispersive elements and an intermediate focus it is possible to eliminate nearly all slit scattering by completely removing $w_{2}$, in which case the energy spread is given by expressions $1 \mathrm{~b}$ and 2c. The thickness of the slits $w_{1}$ can be made just thick enough to degrade the energy of the unwanted portion of the beam sufficiently so that the second dispersive element sweeps it off of the target. This 
results in an extremely clean beam. Case $2 a$ is of interest if $S$ is large: decreasing $w_{1}$ decreases the energy spread on the target, but in the limit of $w_{1}=0$ it reduces only to $w_{2} D_{2}$, and the effect of $\mathrm{P}_{1}$ on resolution is lost. In this case the normal procedure would be to force a smaller $\mathrm{S}$ by placing a slit at the source ${ }^{3}$ ). Note also, that any coherence of the beam through $\mathrm{w}_{2}$ (beam on target) is in large measure lost if $S$ is comparable to $w_{1}$. Because spectroscopic measurements designed to take advantage of the coherence across $w_{2}$ are usually made with $\mathrm{w}_{1}$ large the limit on resolution is determined by $S$. If $S$ is the natural source from the accelerator, and not forced by external slits, then $S$ will depend on beam quality and stability of the accelerator, a change of radial position of $S$ with time due to any number of variables such as rf tuning or magnet instability has the same effect as making $\mathrm{S}$ larger. A change in up- and down-stream position of $\mathrm{S}$ has the effect of changing $M_{1}, M_{2}, D_{1}$ and $D_{2}$.

\subsection{COHERENT SOURCE OF WIDTH $S$}

The resolution can be significantly improved if $S$ can be made coherent. Consider as an example that the energy distribution across $S$ is linear and of magnitude reasonable for cyclotrons, namely:

$\Delta E / E=2 \Delta P / P=2 \Delta r / r$ where $\Delta r=S$, and $r=r_{\mathrm{c}}$,

the instantaneous radius of curvature at the time of extraction.

The degeneracy in energy at the image surfaces $x_{1}$ and $x_{2}$ is removed and it can be shown that the energy spread through slits $\mathrm{w}_{2}$, expressed in terms of resolving power is:

$$
\begin{aligned}
R_{\mathrm{c}}= & E_{0} / \Delta E \\
= & M_{1} M_{2} r_{\mathrm{c}} / w_{2}+M_{2}\left[1+M_{1} /\left(1-n_{1}\right)\right] r_{1} / 2 w_{2}+ \\
& +\left[\left(1+M_{2}\right) /\left(1-n_{2}\right)\right] r_{2} / 2 w_{2} .
\end{aligned}
$$

The advantage to be gained by making the source coherent is readily apparent. The equivalent expression for cases $1 a$ and $2 b$ is:

$$
\begin{aligned}
& R_{\mathrm{i}}=E_{0} / \Delta E \\
& =\left[M_{2} r_{1} \frac{\left(1+M_{1}\right)}{\left(1-n_{1}\right)}+\frac{\left(1+M_{2}\right)}{\left(1-n_{2}\right)} r_{2}\right] / 2\left(w_{2}+S M_{1} M_{2}\right),
\end{aligned}
$$

and the ratio of the resolving powers is $R_{\mathrm{c}} / R_{\mathrm{i}}=\Delta E_{\mathrm{c}}$ (coherent) $/ \Delta E_{\mathrm{i}}$ (incoherent) $=$

$$
\begin{array}{r}
{\left[1+S M_{1} M_{2} / w_{2}\right]\left\{1+2 M_{1} M_{2} r_{\mathrm{c}} /\left[M_{2} r_{1}\left(1+M_{1}\right) /\left(1-n_{1}\right)\right.\right.} \\
\left.\left.+r_{2}\left(1+M_{2}\right) /\left(1-n_{2}\right)\right]\right\} .
\end{array}
$$

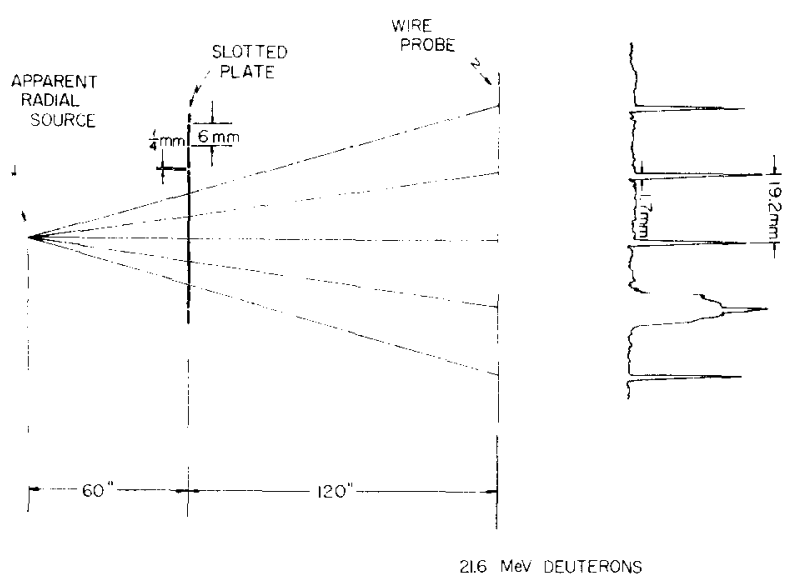

Fig. 6. Geometry used to measure emittance. A trace obtained with the 0.009 " dia. wire probe is shown at the right. The very broad peak results from an indexing hole in one of the slits.

For the case $M_{1}=M_{2}=1$ and $n_{1}=n_{2}=\frac{1}{2}$ this reduces to

$$
R_{\mathrm{c}} / R_{\mathrm{i}}=\left(1+S / w_{2}\right)\left[1+r_{\mathrm{c}} / 2\left(r_{1}+r_{2}\right)\right] .
$$

For the effective source from an isochronous cyclotron to be coherent the following conditions must be met: a) the amplitude of the incoherent radial oscillation must be zero, $b$ ) there must not be precessional mixing so that there is single turn extraction, and c) extraction should be accomplished on advancing phase. While these requirements cannot be met in practice, they serve as a guide in improving the orbit dynamics and stability to optimize the intensity and resolving power.

\section{Source emittance and line-shape}

An important number for high resolution spectroscopy is the brightness* of the source which is directly related to its emittance, or phase-space density. One method used to determine the position, size, and emittance of the source from The University of Michigan 83-inch cyclotron was that of Grunder et al. ${ }^{4}$ ) Measurements were made for deuteron beams of $15 \mathrm{MeV}, 21.8 \mathrm{MeV}$ and $28.9 \mathrm{MeV}$. Briefly, the method consists of placing a carbon plate in the beam duct well outside the fringe field of the main magnet. The plate contains vertical slots of width $0.010^{\prime \prime}$ spaced $0.250^{\prime \prime}$ apart as shown in fig. 6. The images of the slots are observed on a fluorescent screen some 120 inches downstream, and their profile measured with a 0.009 "

\footnotetext{
* Brightness is here defined as $B=I / A_{x} A_{y}$ where $I$ is the intensity (energy per second or number of particles per second times the energy per particle) and $A_{x}$ and $A_{y}$ are the areas of $x$ and $y$ phase space, generally expressed in $\mathrm{mm}$ mrad.
} 
diameter wire probe. The current to the probe as a function of position across the duct was recorded on any $x-y$ plotter. A reproduction of one such graph is also shown in fig. 6. From these data the position of the source was determined to be 60.2 inches upstream from the slotted plate, corresponding to 0.3 inches downstream from the centerline of the dees at a radius of 45 inches from the center of the cyclotron. The radial emittance was determined from the $\theta$ vs $x$ plot (shown in fig. 7 for $15 \mathrm{MeV}$ deuterons). Since $\theta=p_{r} / p$ and $x=\Delta r$ the enclosed area in the figure is the (two dimensional) radial phase space density $p_{r} \cdot r$ to within the factor $p$. The emittance was determined to be $24 \mathrm{~mm}$ mrad for $30 \%$ of the extracted beam which leads to a radial width for the effective source of $0.51 \mathrm{~mm}$.

Such measurements are invaluable in determining the quality of the extracted beam and the properties of the source, and are made not only for all newly developed beams, but also routinely to check on the

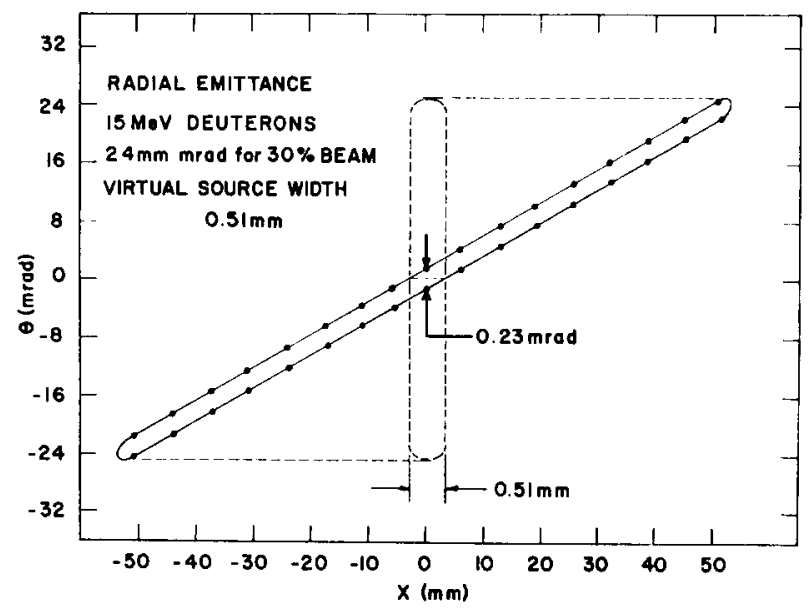

Fig. 7. Radial phase space density.
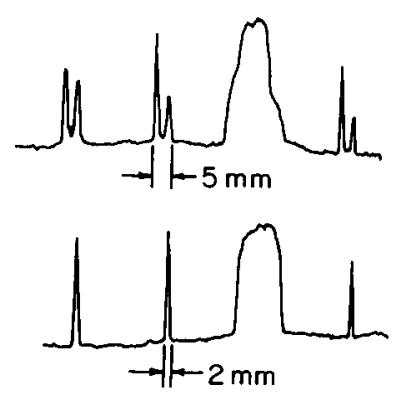

Fig. 8. Intensity vs radial position measured with the wire probe 120 inch downstream of the slotted plate. The upper trace, indicating extraction of two orbits, resulted from slight mistuning of the cyclotron. The broad peaks are caused by the indexing hole. beam quality. Traces resulting from beams of poor and good quality are shown in fig. 8 . The double peaks indicate extraction of beam from more than one turn which results in a wide source $S$ and poor emittance. The usual procedure is to view the image pattern on a fluorescent screen which is adequate to determine that the beam quality is satisfactory over the full range of tuning conditions for which beam enters the scattering chamber. The radial position is essentially independent of deflector voltage, but may vary as much as a few millimeters with $\mathrm{rf}$ frequency and dee voltage. Stability of these tuning parameters is therefore essential for high resolution spectroscopy.

The spread in energy of the beam extracted from the cyclotron is measured by means of the beam preparation magnets. While it may vary considerably with tuning conditions and with the skill of the operator, values of $E / \Delta E=300$ or better can be obtained in routine operation.

The study of the line shape on the focal surface of $\mathrm{P}_{2}$ (the target position) gives useful information on the parameters $S, M_{1}, M_{2}, D_{1}$ and $D_{2}$.

The actual shapes were measured using as a current probe a $0.009^{\prime \prime}$ diameter tungsten wire driven normal to the optic axis and recording the current as a function of position on an $x-y$ plotter. The measured curves together with the computed line-shapes, after folding in the wire diameter, are shown in fig. 9. The slopes of the lines are a measure of the radial width $S$ of the effective source, and from these measurements the value of $S$ was determined to be $0.46 \mathrm{~mm}$, in good agreement with the values determined by the other techniques.

The value of $S$ may also be determined from measurements using the three reaction products analysis magnets. In the initial design ${ }^{1}$ ) of the total spectrometer the sum of the dispersions of the three analyzer magnets was made equal but opposite to the sum of the dispersions of the two beam preparation magnets. It is easily shown that for a coherent beam transported through the five magnets (with the analyzer magnets set at $0^{\circ}$ ), the width of the image at the final image

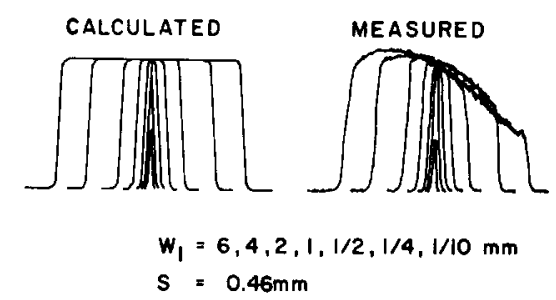

Fig. 9. Calculated and measured line shapes at the focal surface of $\mathrm{P}_{2}$, the center of the scattering chamber, for several values of $w_{1}$. 
surface is

$$
\begin{array}{r}
Y=S\left\{1-\left[\left(1-n_{\mathrm{B}}\right) /\left(1-n_{\mathrm{A}}\right)\right]\left[\left(1+\left|M_{\mathrm{A}}\right|\right) /\left(1+\left|M_{\mathrm{B}}\right|\right)\right] .\right. \\
\left.\cdot \sum r_{\mathrm{A}} / \sum r_{\mathrm{B}}\right\},
\end{array}
$$

and is independent of the width of slits $w_{1}$ and $w_{2}$. Since for this system $n_{\mathrm{A}}=n_{\mathrm{B}}=\frac{1}{2}, M_{\mathrm{A}}=M_{\mathrm{B}}=-1$, and $\Sigma r_{\mathrm{A}}=\Sigma r_{\mathrm{B}}=400 \mathrm{~cm}$, then $Y=0$. Because $S$ is not coherent the width of the final image $Y$ is not zero but is the width of the effective source from the cyclotron. Using a fluorescent screen, this was determined to be $(0.5 \pm 0.1) \mathrm{mm}$, in good agreement with the slotted plate measurements.

\section{Determination of image surfaces and aberrations}

The image distance of the beam preparation system is readily determined using the wire probe technique by measuring the line-shape as a function of the position up and down stream. The results of a series of such measurements are shown in fig. 10. The field index $n$ for each magnet and the location of the magnets with respect to the cyclotron were adjusted to put the image at the center of the scattering chamber.

The same technique has also been used to determine the image distance of each of the three reaction products analyzer magnets, the shape of each image surface, and the magnitude of the ion-optical aberrations. A slit placed at the image position of the beam preparation system (the center of the scattering chamber) serves as a source for the analyzer magnets which are set at $0^{\circ}$ with respect to the incident beam. The up- and downstream position of the best image of the first magnet was determined as a function of position transverse to the optic axis, the transverse

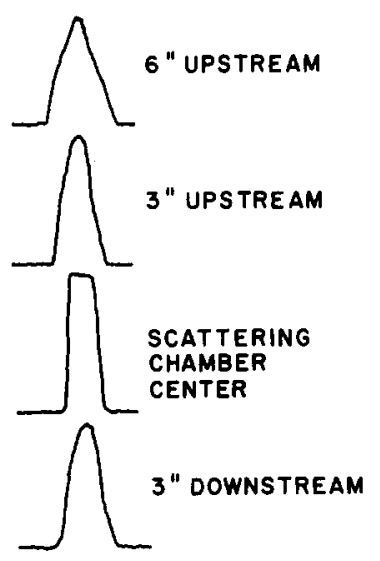

Fig. 10. Line-shape vs up- and down-stream position in the scattering chamber. The line-shape at the scattering chamber center agrees closely with the calculated shape. position of the beam being varied by varying the magnetic field. The shape of the image surface was mapped in this way; since the magnitude of the magnetic field is measured with a NMR probe, the dispersion of the magnet in $\mathrm{kHz} / \mathrm{mm}$ is also determined. Similar measurements were made to determine the image surfaces of the second and third magnets. The measurements also serve to calibrate the field (as measured by the NMR) of each magnet and ensure that the particles traverse the magnets symmetrically.

As indicated in sec. 3 the width $Y$ of the image at the focal surface of the third analyzer magnet (AM3) should be independent of the widths of slits $w_{1}$ and $w_{2}$. The intensity of the image should be proportional to $w_{1}$ (assuming $w_{2}=\infty$ ). The line shape measured at the focal surface of AM3 for various values of $w_{1}$ from $0.1 \mathrm{~mm}$ to $6.0 \mathrm{~mm}$ ( $w_{2}$ removed) is shown in fig. 11. It should be noted that as $w_{1}$, and therefore the beam current and the width of the beam spot in the scattering chamber, are increased by a factor of 60 , the profile of the image remains essentially constant. The lower limit on width, $1.9 \mathrm{~mm}$, (corresponding to $1.15 \mathrm{~mm}$ normal to the optic axis) is due mainly to the source. This was confirmed by a subsequent direct measurement at the machine exit which, for the tuning condition of this particular run, yielded $S=1.4 \mathrm{~mm}$, in good agreement with the above value. This again emphasizes the importance of the contribution of the source to resolution. We are presently developing instrumentation to allow continuous monitoring of the source size and position during experimental runs.

The image surfaces determined using the direct beam and the wire probe correspond to those that

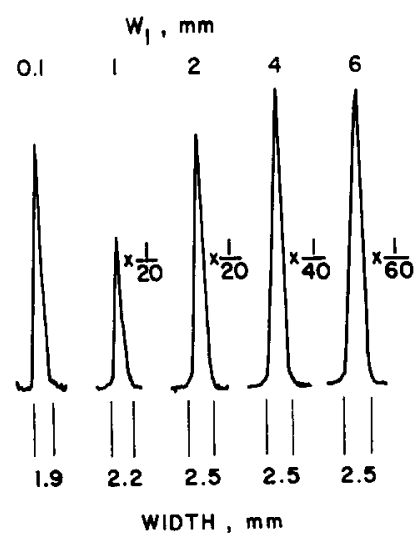

Fig. 11. Line-shapes at the focal surface of the third analyzer. Note that the intensities are essentially proportional to $w_{1}$. The peak for $w_{1}=6 \mathrm{~mm}$ is very nearly 60 times more intense than for $w_{1}=0.1 \mathrm{~mm}$. 
would result from a nuclear reaction on an infinite mass target; the focal surface for reaction products will vary depending on the kinematics of the particular reaction $^{5}$ ). With our system, the kinematic focal surface is inside of the infinite mass surface, the exact location depending of the value of $(1 / 2 E)(\mathrm{d} E / \mathrm{d} \Psi)$ which for the reaction $\mathrm{A}(\mathrm{a}, \mathrm{b}) \mathrm{B}$ is $\approx \sqrt{ }\left(M_{\mathrm{a}} M_{\mathrm{b}} / M_{\mathrm{A}} M_{\mathrm{B}}\right)$ $\sin \Psi$. The exact value of this function also depends on the reaction $Q$-value and is normally computed as a part of a more general kinematics program. The focal surfaces of the three magnets, as a function of the parameter $(1 / 2 E)(\mathrm{d} E / \mathrm{d} \Psi)$ have been measured using actual reaction products from a variety of finite mass targets.

It should be noted that while the dispersions of a preparation and an analysis system do not have to be exactly matched to permit cancellation since proper choice of the target angle will, in principle, allow a beam spot of finite width to be completely compensated, in practice, in order to avoid extreme target angles, the match must be reasonably close. Fig. 12 illustrates the cancellation obtained using two of the three analyzers. The peaks shown result from $21.6 \mathrm{MeV}$ deuterons scattered elastically at $30^{\circ}$ from a $300 \mu \mathrm{g} / \mathrm{cm}^{2} \mathrm{~Pb}$ target set at minus $22^{\circ}$ to cancel the effect of the beam width. The width of the peaks is due primarily to the target thickness. In this case because of the dispersion mismatch it is not possible

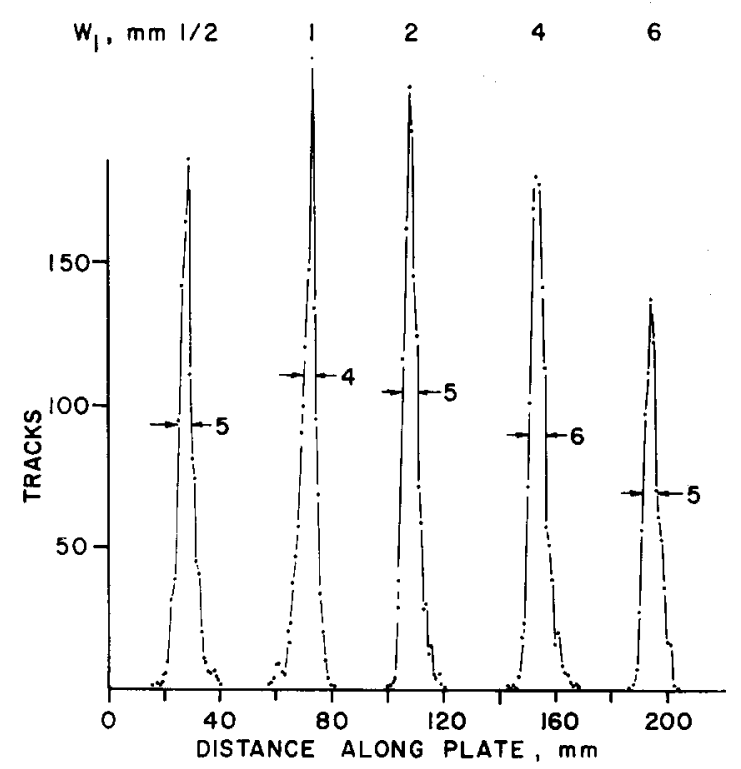

Fig. 12. Line-shapes at the focal surface of the second analyzer. Exposures have been adjusted to yield approximately the same number of tracks in each peak, and thus vary by a factor of 12 as $w_{1}$ is opened from $1 \mathrm{~mm}$ to $6 \mathrm{~mm}$. to compensate for both finite beam spot and target thickness ${ }^{5}$ ). As the slit $w_{1}$ is opened from $\frac{1}{2} \mathrm{~mm}$ to $6 \mathrm{~mm}$, increasing the current on the target and the spot width by a factor of 12 , the resolution remains essentially unchanged.

\section{Resolution and brightness}

In practice the resolution that it is feasible to obtain in a given high resolution experiment is limited by the magnitude and quality of the current available in the scattering chamber and this is limited by the brightness of the source $\mathrm{S}$. The brightness is determined mainly by the magnitude and quality of the current available in the cyclotron just before extraction; for isochronous cyclotrons the magnitude is limited either by the brightness of the ion source or by space charge.

With properly designed hooded arc sources ${ }^{6}$ ) the current is generally limited by space charge for protons and deuterons while for ${ }^{3} \mathrm{He}$, alpha particles, and heavier ions, the current may be limited by the ion source because of the greater power necessary to maintain the density of the ionization in the arc plasma and the fact that a small amount of impurity gas, particularly $\mathrm{H}, \mathrm{D}$, or $\mathrm{O}$, "poisons" the arc by effectively preventing ionization of the helium.

The space charge limit has been calculated ${ }^{7}$ ) and found to be in good agreement with measurements on a variety of cyclotrons*. The limitation generally develops in the first few turns where the total axial focusing force is weakest, corresponding to the minimum frequency of the axial oscillation $v_{z}$. It can be shown ${ }^{7}$ ) that to good approximation the limit is

$$
I(\mathrm{~A})=h \varepsilon_{0} v_{z}^{2}(\Delta \Phi / 2 \pi)(\Delta E / e) \omega,
$$

where $h$ is the aperture or height of the dee, $(\Delta \Phi / 2 \pi)$ is the azimuthal extent of the beam (the duty cycle), $(\Delta E / e)$ is the energy gain per turn expressed in electron volts, $\varepsilon_{0}$ is the dielectric constant for free space and $\omega$ is the angular frequency of the rf, all in MKS units. For The University of Michigan cyclotron, for example, $v_{z_{\min }} \approx 0.16$ at $r \approx 8^{\prime \prime}$ which corresponds to $E_{\max } / 20$. For the dee aperture of $h=3 \times 10^{-2} \mathrm{~m}$, an energy gain per turn of $100 \mathrm{keV}$ and $\omega \approx 50 \times 10^{6}$, the space charge limited current is of the order of $40(\Delta \Phi / 2 \pi) \mathrm{mA}$. For a duty cycle $(\Delta \Phi / 2 \pi)$ of $\frac{1}{50}$, $\left(\approx 7^{\circ}\right.$ phase width) the space charge limited current is about $800 \mu \mathrm{A}$, while for a duty cycle of $\frac{1}{10}\left(36^{\circ}\right.$ phase

* For example, the 88-inch at University of California (Berkeley), the 57-inch at Michigan State University, and the 83-inch at The University of Michigan. We are indebted to B. G. Harvey and $\mathbf{H}$. G. Blosser for providing us with their data. 
width) the limit is $4 \mathrm{~mA}$. In general phase widths larger than $7^{\circ}-10^{\circ}$ do not increase the useful current to the scattering chamber, for reasons discussed below. It might be noted that flat-topping the rf circumvents this limitation and promises an order of magnitude increase in the useful current.

The quality of the circulating beam just prior to extraction depends on the brightness of the ion source, the build-up of incoherent betatron osillations, and on such factors as precessional mixing. The brightness of the ion source is in general adequate. Measurements $^{8}$ ) on a hooded source indicate a value of $46 \pi$ $\mathrm{mm} \mathrm{mrad}$ for $38 \mathrm{~mA}$ of protons at $200 \mathrm{kV} \mathrm{dc}$. When extrapolated to $50 \mathrm{MeV}$ protons, this corresponds to an emittance of approximately $10 \mathrm{~mm} \mathrm{mrad}$, and a current of $760 \mu \mathrm{A}$ for a duty cycle of $7^{\circ}$. The amplitude of the incoherent oscillations depends on a variety of factors such as space charge, traversal of the first $v_{\mathrm{r}}=1$ resonance, and imperfections in the magnetic field. Precessional mixing is directly related to the coherent radial oscillation amplitude and the duty cycle $^{9}$ ). When a coherent radial osillation is present the particles precess around the magnetic center of the guide field, the number of particle turns for a full precessional period being $1 /\left(v_{\mathrm{r}}-1\right)$. Particles accelerated at slightly different $\mathrm{rf}$ phase have different energy gains per turn and thus make a different number of turns in reaching the extraction radius; therefore they make a different number of radial precessions. If the number of precessional periods differ by one then complete precessional mixing occurs, and the coherent radial oscillation amplitude becomes incoherent. In many isochronous cyclotrons the value of $v_{\mathrm{r}}$ is near 1.02 , so that the number of particle turns per precessional period is of the order of 40 to 50 . Thus, if the total number of particle turns a)

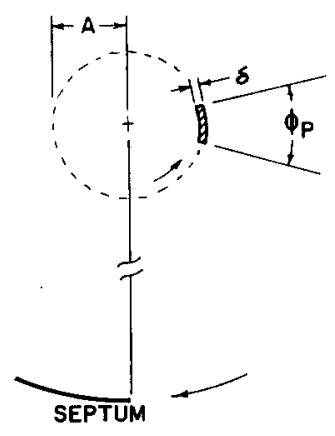

b)

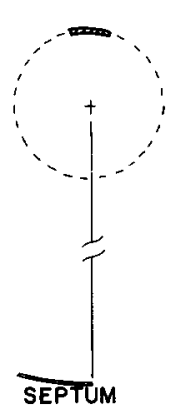

Fig. 13. Effect of orbit centers on $R_{e}$. to extraction radius is 250 , the number of precessional turns will be $250\left(v_{\mathrm{r}}-1\right) \approx 5$. In general single turn extraction requires that the phase width be of the order of $7^{\circ}$ and the incoherent amplitude must be small.

Assume that the amplitude $A$ of the coherent oscillation is $3 \mathrm{~mm}$ (a reasonably small amplitude) and that the amplitude $\delta$ of the incoherent oscillation is zero (see fig. 13a). Then the difference in radius from the instantaneous orbit centers to the septum can be as large as $A \phi_{\mathrm{p}}$ which for $\phi_{\mathrm{p}}=30^{\circ}$ corresponds to $1.5 \mathrm{~mm}$, and for an extraction radius of $1000 \mathrm{~mm}$ this corresponds to a variation in energy of $0.3 \%$ or $R_{\mathrm{e}}=333$. If the extractor system will accept the full $2 A=6 \mathrm{~mm}$ then $R_{\mathrm{e}} \approx 167$. However, if stability of the cyclotron components is sufficiently good to hold the instantaneous centers fixed near the positions shown in fig. $13 \mathrm{~b}$, then $R_{\mathrm{e}}$ can be as large as 5000 . The problems in obtaining this kind of stability are severe. In general the incoherent amplitude $\delta$ will not be zero and will add to $A$ and reduce $R_{\mathrm{e}}$.

The considerable advantages of careful adjustment of the internal orbits include reduced power dissipation on the septum, reduced background radiation, and good control of the quality and position of the effective source.

If the phase width is limited only by the arc tower and puller geometry (for the usual geometry this will be about $30^{\circ}$ ), there will be complete precessional mixing and for an amplitude of radial oscillation of $3 \mathrm{~mm}, \Delta r=6 \mathrm{~mm}$ and $\Delta r / r_{\mathrm{c}}=6 \times 10^{-3}$ and $\mathrm{R}_{\mathrm{e}}=80$. If the extractor channel transmits this, then for $E / \Delta E=8 \times 10^{3}$ in the scattering chamber the current in the scattering chamber will be reduced by a factor of 100 without regard to emittance. In general when the internal beam quality is poor, it is the power dissipation on the septum that limits the beam. If the extractor efficiency is $50 \%$ and the septum can dissipate $3 \mathrm{~kW}$, then for $50 \mathrm{MeV}$ particles, the maximum current that can be extracted is about $60 \mu \mathrm{A}$.

The current of given quality available at the target in the scattering chamber after extraction and beam analysis can be estimated $\left.{ }^{10}\right)$. Assume that the beam current $I(\Delta \Phi / 2 \pi)$ is extracted from the cyclotron with an extraction efficiency $\varepsilon$, that its radial phase-space density is $Q \mathrm{~mm} \operatorname{mrad}$ for $p \%$ of the beam with an energy spread $\Delta E_{\mathrm{e}}$ given by $R_{\mathrm{e}}=E / \Delta E_{\mathrm{e}}$. The current in the scattering chamber of quality $q$ and resolution $R=E / \Delta E$ will be

$$
I_{\mathrm{sc}}=I(\Delta \Phi / 2 \pi) \varepsilon p(q / Q) R_{\mathrm{e}} / R .
$$

Assume as "typical for isochronous cyclotron operation the values $Q=50 \mathrm{~mm} \operatorname{mrad}, p=0.80, \varepsilon=0.5$, 
and $R_{\mathrm{e}}=3 \times 10^{2}$ and that the requirement on the beam in the scattering chamber for high resolution measurements is $R \approx 10^{4}$ with a quality $q=10 \mathrm{~mm}$ mrad (approximately $0.6 \mathrm{~mm}$ wide spot with one degree divergence), then

$$
I_{\mathrm{sc}}=I(\Delta \Phi / 2 \pi) \times 2.4 \times 10^{-4} .
$$

If $I(\Delta \Phi / 2 \pi) \approx 800 \mu \mathrm{A}$, then $I_{\mathrm{sc}} \approx 200 \mathrm{nA}$. Thus the current to the scattering chamber is limited not only by the stringent requirements on $R$ and $q$, but also by the fact that the useful beam to the scattering chamber is contained in a small phase width during acceleration, and increasing the duty cycle $(\Delta \Phi / 2 \pi)$ will not in general increase the current to the scattering chamber.

\section{Calibration of the spectrometer}

For precision nuclear spectroscopy it is essential that the magnetic fields rather than the current of each of the magnets be regulated and that hysteresis and saturation effects, hopefully small, are known. The magnetic fields of the cyclotron magnet and each of the five spectrometer magnets are regulated* using NMR fluxmeters; the same fluxmeters are also used for determining the magnitudes of the fields. Because the fields fall-off with radius $\left(n=\frac{1}{2}\right)$, the NMR probes are located in radius near the inside edge of the gap at the turn-over point where the field is a maximum and has a horizontal tangent, and in azimuth near the entrance where, because of the divergence of the beam, it does not reduce the solid angle.

The important considerations for spectroscopy are 1) the absolute calibration of the average value of the field at all fields, 2) the reproducibility of the fields from day-to-day, and 3) differential hysteresis along the magnetic path.

The second beam preparation magnet has been calibrated in terms of the $T=\frac{3}{2}$ isobaric analogue resonance in ${ }^{13} \mathrm{~N}$ which occurs at a laboratory energy of $14.233 \mathrm{MeV}$ in the ${ }^{12} \mathrm{C}(\mathrm{p}, \mathrm{p})$ excitation function $\left.{ }^{11}\right)$. The resonance has been measured at $165^{\circ}$ several times $^{\dagger}$, first in May, 1966 and most recently in June, 1969. A typical result is shown in fig. 14. The width of the resonance, peak to valley, is nearly $3 \mathrm{keV}$ and the peak to valley ratio is approximately 1.8 . These data were taken with a $40 \mu \mathrm{g} / \mathrm{cm}^{2}(1.4 \mathrm{keV})$ carbon target and

\footnotetext{
* The details of the regulator circuits will be reported in a separate paper.

$\dagger$ We are much indebted to our colleague W. S. Gray for carrying out several of these measurements.
}

an energy spread of the incoming beam on the target of $1.8 \mathrm{keV}$. The points plotted with circles were taken by varying the magnetic field of the second beam preparation magnet, and those plotted with $X$ were taken keeping the magnetic field constant and varying the dc potential of the target. The position of the resonance is reproducible within the accuracy of the measurement $(\approx 200 \mathrm{~V})$ independently of the past history of the magnetic field. No shift in position as large as $200 \mathrm{~V}$ was detected when the field was brought from zero to the resonance value, or from zero to full excitation then down to the resonance value, or with small changes (corresponding to $200 \mathrm{keV}$ ) across the resonance. Thus, it is concluded that the average field is reproducible to within 1 part in 12000 and any uncertainties due to hysteresis effects are less than $1 \mathrm{keV}$.

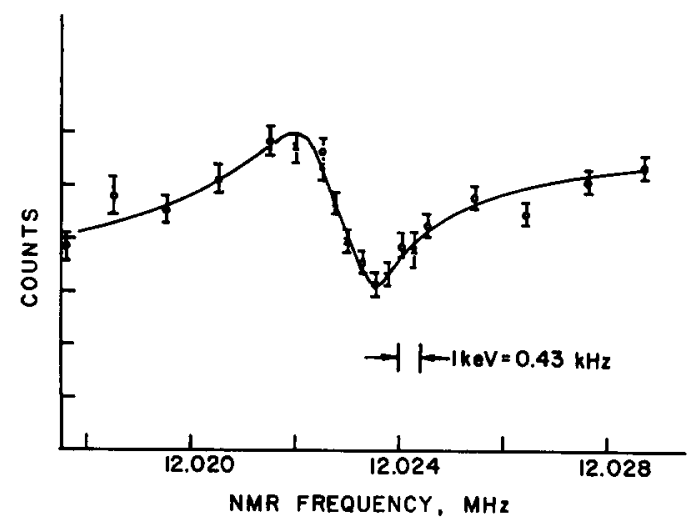

Fig. 14. The ${ }^{13} \mathrm{~N} T=\frac{3}{2}$ resonance at $E_{\mathrm{p}}=14.233 \mathrm{MeV}$ in the excitation function for ${ }^{12} \mathrm{C}(\mathrm{p}, \mathrm{p})$.

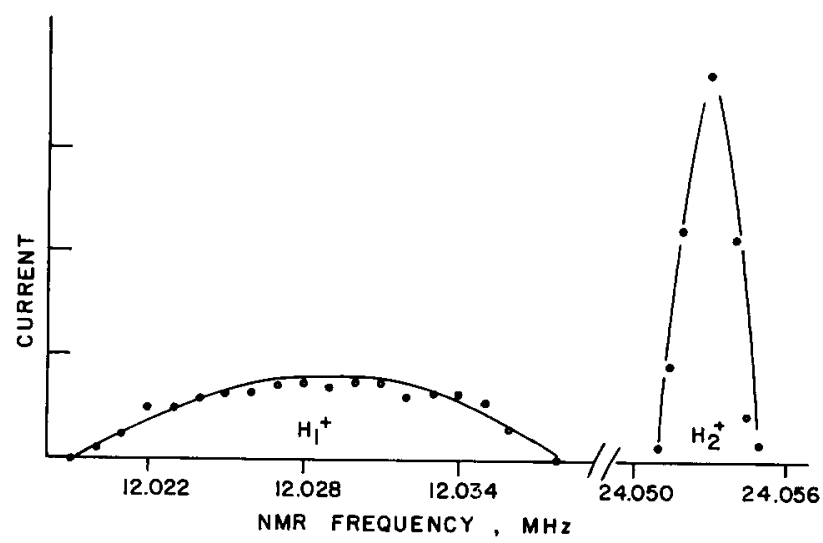

Fig. 15. Current vs the NMR frequency of the second beam preparation magnet, for $\mathrm{H}_{1}^{+}$and $\mathbf{H}_{2}^{+}$ 
The three reaction products analysis magnets were calibrated using this well defined beam. Thus, the entire system is calibrated at one energy in terms of the $T=\frac{3}{2}$ resonance in ${ }^{13} \mathrm{~N}$.

To determine the linearity of the magnet system with excitation well defined beams at higher energies are needed. The $\mathrm{H}_{2}^{+}$ion consisting of two protons of essentially equal energy plus an electron would appear to offer a calibration at twice the energy of $\mathrm{H}_{1}^{+}$at the $T=\frac{3}{2}$ resonance (corrected of course for the electron mass). Unfortunately, due to the 2.65 electron volt dissociation energy of the $\mathrm{H}_{2}^{+}$molecule ion, the spread in kinetic energy of the two protons on break-up of the $\mathrm{H}_{2}^{+}$is approximately $42 \mathrm{keV}$ which is more than enough to mask the resonance ${ }^{12}$ ). However, while the resonance cannot be detected with $\mathrm{H}_{2}^{+}$, a calibration can be made by adjusting the two beam preparation magnets to transport $\mathrm{H}_{2}^{+}$to the scattering chamber through small slit openings. A thin stripping foil placed at the intermediate focus of the two-magnet system dissociates the $\mathrm{H}_{2}^{+}$into $\mathrm{H}_{1}^{+}+\mathrm{H}_{1}^{+}$.

The field of the second magnet must then be reduced from that corresponding to $28.474 \mathrm{MeV}$ to $14.233 \mathrm{MeV}$ to bring the $\mathrm{H}_{1}^{+}$into the scattering chamber. The relation between the two energies is well defined, and this serves to calibrate the magnet system at essentially twice the energy of $14.233 \mathrm{MeV}$. The currents to the Faraday cup as a function of the NMR frequency of the second magnet (which is proportional to the magnetic field and hence to momemtum) is shown in fig. 15 for $\mathrm{H}_{2}^{+}$and $\mathrm{H}_{1}^{+}$. The line shape for $\mathrm{H}_{2}^{+}$is that expected from the geometry, as discussed in sec. 3. The full width of the $\mathrm{H}_{1}^{+}$curve is $18.7 \mathrm{kHz}$ which, when corrected for target thickness and the energy spread in the target, leads to the value of $\Delta E=42 \mathrm{keV}$ as the spread in energy resulting from dissociation. It is interesting to note that this corresponds to a potential energy of the repulsive state of the $\mathrm{H}_{2}^{+}$ of 7.8 e.v. Further, the ratio of the intensities measured using first the $\mathrm{H}_{2}^{+}$beam then the $\mathrm{H}_{1}^{+}+\mathrm{H}_{1}^{+}$beam suggest that the break-up channel is $\mathrm{H}_{2}^{+} \rightarrow \mathrm{H}_{1}^{+}+\mathrm{H}_{1}^{+}$, with essentially none of the $\mathrm{H}_{2}^{+} \rightarrow \mathrm{H}_{1}^{0}+\mathrm{H}_{1}^{+}$component.

If the magnetic field scaled accurately with the NMR frequency, the ratio of the frequencies should be

$$
\begin{aligned}
\frac{f\left(H_{2}^{+}\right)}{f\left(H_{1}^{+}\right)}=\frac{B \rho\left(H_{2}^{+}\right)}{B \rho\left(H_{1}^{+}\right)}=\frac{m\left(H_{2}^{+}\right)}{m\left(H_{1}^{+}\right)}=\frac{2 m_{\mathrm{p}}+m_{\mathrm{e}}}{m_{\mathrm{p}}} \\
=\left(2+\frac{m_{\mathrm{c}}}{m_{\mathrm{p}}}\right)=2+5.45 \times 10^{-4} .
\end{aligned}
$$

Experimentally, when the $\left(\mathrm{H}_{1}^{+}\right)$frequency is corrected for the energy loss in the carbon stripping foil $\left(40 \mu \mathrm{g} / \mathrm{cm}^{2}\right)$, the difference of the calculated and measured values is $11.2 \mathrm{kHz}$ at $24.06 \mathrm{MHz}$ or the deviation from perfect scaling of the magnetic field corresponding to $14.2 \mathrm{MeV}$ protons and $28.4 \mathrm{MeV}$ protons is $\Delta f \mid f=1 / 2140$ or less than $0.05 \%$. The scaling factor for other and higher fields will be measured using ${ }^{3} \mathrm{He}^{+}$and ${ }^{3} \mathrm{He}^{++}$where the energy spread due to molecular dissociation does not enter.

\section{References}

1) J. Bardwick, J. M. Lambert and W. C. Parkinson, Nucl. Instr. and Meth. 18, 19 (1962) 105; W. C. Parkinson and J. Bardwick, Bull. Am. Phys. Soc. 13 (1968) 557.

2) D. L. Judd, Rev. Sci. Instr. 21 (1950) 213.

3) D. R. Bach, W. J. Childs, R. W. Hockney, P. V. C. Hough and W. C. Parkinson, Rev. Sci. Instr. 27 (1956) 516; see also, P. A. Reeve, Nucl. Instr. and Meth. 67 (1969) 13.

4) H. A. Grunder, F. B. Selph and H. Atterling, Proc. Intern. Conf. Sector-focused cyclotrons, CERN 63-19 (1963) p. 59.

5) B. Sjögren, Nucl. Instr. and Meth. 7 (1960) 76.

6) R. S. Livingston and R. J. Jones, Rev. Sci. Instr. 25 (1954) 552; M. L. Mallory and H. G. Blosser, Trans. IEEE NS-13 (1966) 163.

7) See for example, H. Blosser and M. M. Gordon, Nucl. Instr and Meth. 13 (1961); K. R. Mackenzie, Nucl. Instr. and Meth. 31 (1964) 139; J. D. Lawson, Nucl. Instr. and Meth. 34 (1965) 173, also 64 (1968) 214: J. Rainwater, Rev. Sci. Instr. 37 (1966) 262; Martin Reiser, Trans. IEEE NS-13 (1966) 171 (Proc. Intern. Conf. Isochronous cyclotrons, Gatlinburg).

8) H. G. Blosser and M. M. Gordon, Nucl. Instr. and Meth. 13 (1961) 101; A. McKeener and B. Yokosawa, Rev. Sci. Instr. 33 (1962) 7; R. S. Livingston, Proc. Intern. Conf. Sectorfocused cyclotrons and meson factories, CERN 63-19 (1963) p. 1; M. L. Mallory and H. G. Blosser, Trans. IEEE NS-13 (1966) 163 (Proc. Intern. Conf. Isochronous cyclotrons, Gatlinburg).

9) See, H. A. Grunder, Trans. IEEE NS-13 (1966) 371 (Proc. Intern. Conf. Isochronous cyclotrons, Gatlinburg).

10) See also, B. L. Cohen, Rev. Sci. Instr. 33 (1962) 85.

11) G. Temmer, Nuclear Structure (International Atomic Energy Agency, Vienna, 1968) p. 258; J. B. Marion, Rev. Mod. Phys. 38 (1966) 660.

12) K. H. Purser, P. H. Rose, N. B. Brooks, R. P. Bastide and A. B. Wittkower, Phys. Letters 6 (1963) 176. 